\title{
Nonlinear Space Charge Wave Theory of Distortion in a Klystron
}

\author{
John G. Wöhlbier, Member, IEEE, and John H. Booske, Senior Member, IEEE
}

\begin{abstract}
We present a new view of nonlinear distortion in a klystron based on an analytically solvable nonlinear Eulerian model. The nonlinear contributions to the analytic solutions for the beam modulations are "nonlinear space charge waves" in the sense that they are produced by the nonlinear mixing of the linear space charge waves. For a single-frequency input, amplitude and phase distortion are shown to be results of "self-intermodulation" at the drive frequency, or mixing of harmonic distortions with the fundamental. The self-intermodulation contributions add out of phase with the linear space charge waves to produce gain compression and phase distortion. By comparing the results to a conventional large-signal Lagrangian model we find this physical picture is accurate for drive levels up to $1.2 \mathrm{~dB}$ of gain compression. For a two-frequency input we predict the third-order intermodulation distortion generation and suppression with the nonlinear space charge wave theory.
\end{abstract}

Index Terms-Amplitude distortion, intermodulation, klystron, linearization, phase distortion.

\section{INTRODUCTION}

$\mathbf{U}$ NDERSTANDING the generation and mitigation of harmonic and intermodulation distortion in linear-beam microwave vacuum electronic devices (MVEDs) is currently an active area of research, e.g., [1]-[4]. The use of modern largesignal MVED codes in the design process (e.g., [3], [5], and [6]) has resulted in improved device performance, including enhanced performance in the nonlinear regime of the device transfer characteristics. Thus, the development of these codes and their incorporation into design and analysis of microwave vacuum electronic devices has been and will continue to be beneficial.

However, in efforts to understand device physics through analysis and simulation that parallel modern MVED code development, approximate analytic solutions of Lagrangian formulations to date [7], [8] have not yielded direct physical insights. In contrast, analytic treatments of nonlinear Eulerian models do yield direct physical insights. We have recently

Manuscript received July 13, 2004; revised October 20, 2004. The work of J. G. Wöhlbier was supported in part by the Department of Energy, Los Alamos National Laboratory, Threat Reduction Directorate, as an Agnew National Security Postdoctoral Fellow. This work was supported in part by the U.S. Air Force Office of Scientific Research (AFOSR) under Grant 49620-00-1-0088 and in part by the Deputy Under Secretary of Defense for Science and Technology under the Innovative Microwave Vacuum Electronics Multidisciplinary University Research Initiative program, managed by the AFOSR under Grant F49620-99-1-0297. The review of this paper was arranged by Editor W. L. Menninger.

J. G. Wohlbier is with Continuum Dynamics Group (CCS-2), Los Alamos National Laboratory, Los Alamos, NM 87545 USA.

J. H. Booske is with the Department of Electrical and Computer Engineering, University of Wisconsin, Madison, WI 53706 USA.

Digital Object Identifier 10.1109/TED.2005.845865 reported on a number of topics where the analytic solution to an Eulerian model has yielded insights into nonlinear traveling wave tube (TWT) behavior [9]-[14]. Note that without special techniques [15] Eulerian models will strictly be limited to inputs where electron overtaking does not occur. However, as we show in this paper, for inputs where the amount of electron overtaking is small, the Eulerian and Lagrangian models are in reasonable agreement.

In this paper we apply the modeling and analysis methods of [9]-[12] to a modulated electron beam as found in a two cavity klystron. In the context of the modulated electron beam the analytic treatment of the nonlinear Eulerian model results in a "nonlinear space charge wave" theory. That is, nonlinear evolution of electron beam velocity and density are described in terms of nonlinear mixing of the space charge waves of linear theory [16], [17]. We apply this description to gain insights into transfer characteristic distortions (AM/AM and AM/PM) and intermodulation distortion generation and suppression. In particular, amplitude and phase distortion are explained in terms of a "self-intermodulation" process where second harmonic beam distortions nonlinearly mix with linear space charge waves to produce third-order intermodulation (3IM) distortions at the drive frequency. These 3IM distortions are phased such that they subtract from the linear space charge waves, and this results in amplitude and phase distortion relative to the linear behavior. For a two tone input we show that the generation of 3IM distortions (e.g., $2 f_{a}-f_{b}$ ) comes from mixing second-order distortions (sum frequency and harmonics) with the linear space charge waves. Furthermore, suppression of the generated 3IM by injecting a signal at the 3IM frequency [4] is approximately described as a superposition of the nonlinearly generated 3IM and linear space charge waves due to the (small) injected 3IM. This view sheds light on the operation of predistortion equalizers since they inherently work on the principle of 3IM injection [18].

In Section II we discuss the models used to study the distortion problem, referring the reader to [19, App. E] for detailed model equations, and the Appendix of this paper for discussions of analytic solutions to the Eulerian model. In Section III we use the Eulerian model to study a single-frequency input. The Eulerian model is first compared to the conventional Lagrangian model to determine validity of the Eulerian model for an input level of practical interest, i.e., an input such that the output shows more than $1 \mathrm{~dB}$ of gain compression. After the validation we compare simulation and analytic predictions of harmonic, amplitude, and phase distortion. In Section IV we consider the two-frequency input problem. The Eulerian model is first compared to the conventional Lagrangian model to determine validity for a case that has been studied experimentally 
[4], then analytic solutions of nonlinear space charge waves are compared to simulations of the Eulerian model for insights into the mechanisms of distortion generation and mitigation. The paper is concluded in Section V.

\section{MODELS}

Since the TWT and klystron are both linear beam devices, they share the same nonlinear electron beam description, and hence the methodology developed in [9]-[12] may be applied to describe the beam modes in a klystron. The modulated electron beam models used in this paper describe the evolution of the space charge field and the electron beam evolution. The simplified multifrequency spectral Eulerian klystron (S-MUSEK) model represents field and beam quantities spectrally, i.e., $\tilde{E}_{\ell}(z), \tilde{v}_{\ell}(z)$, and $\tilde{\rho}_{\ell}(z)$ represent the space charge field, beam velocity modulation, and beam density modulation at frequency $f_{\ell} \omega_{0}$ respectively, where $z$ is the axial coordinate along the length of the device. For comparison purposes we also consider a conventional "disk" model [20], [21], Lagrangian klystron equations (LAKE), in which the space charge field is still described spectrally with $\tilde{E}_{\ell}$, but the beam evolution is computed by following one period of "disks." We consider only velocity modulated beams where the velocity modulation has a discrete spectrum. See [19] for details of the models. ${ }^{1}$

The S-MUSEK model is approximate and will not agree quantitatively with LAKE results. Furthermore, in its present Eulerian form it is not strictly valid for drive powers when electron overtaking occurs. However, in Sections III and IV we show that for drives such that mild electron overtaking occurs, the numerical and analytical solutions to S-MUSEK are quite close to LAKE predictions. Therefore, we can be confident that the physics in S-MUSEK is correct for the inputs we consider.

The S-MUSEK model has a desirable feature in that it is analytically solvable. The approximate analytic solution of the S-MUSEK model is given in the Appendix. One finds that the solution for a state variable at a particular frequency $f_{\ell} \omega_{0}$ is a sum of complex exponential modes, e.g.

$$
\tilde{\rho}_{\ell}(z)=\left\{\sum A_{\mathrm{dr}} e^{i \gamma_{\mathrm{dr}} z}+\sum A_{\mathrm{nl}} e^{i \gamma_{\mathrm{nl}} z}\right\} e^{i f_{\ell} \omega_{0}\left(\left(z / u_{0}\right)-t\right)}
$$

where $u_{0}$ is the dc electron beam velocity, the subscript " $\mathrm{dr}$ " refers to driven modes, and the "nl" subscript refers to modes arising from nonlinear interactions. For example, the driven modes are the slow and fast space charge waves of conventional klystron theory, and the nonlinear modes are generated by mixing of the driven space charge waves.

\section{Single-FrEQUENCY InPUT}

In this section, we consider a single-frequency velocity modulation. We explore the nonlinear space charge wave description of harmonic distortion, and amplitude and phase distortion at the fundamental. The beam parameters used are given in Table I. $R_{\mathrm{sc}}$ in Table I is related to the space charge reduction factor $R$ of [22] by $R_{\mathrm{sc}}=R^{2}$.
TABLE I

PARAMETERS AND DERIVED QUANTITIES FOR THE BEAM IN [4]

\begin{tabular}{lcr}
\hline \hline Description & Symbol & Value \\
\hline beam voltage & $V_{0}$ & $6.0(\mathrm{kV})$ \\
beam current & $I_{0}$ & $0.6(\mathrm{~A})$ \\
beam radius & $r_{b}$ & $0.783(\mathrm{~mm})$ \\
quarter plasma wavelength & $L=\lambda_{q} / 4$ & $6.21(\mathrm{~cm})$ \\
space charge & $R_{\mathrm{sc}}$ & 0.01 \\
reduction factor & $u_{0}=\sqrt{\frac{2 e V_{0}}{m_{e}}}$ & $4.59 \times 10^{7}(\mathrm{~m} / \mathrm{s})$ \\
\hline dc beam velocity & $\rho_{0}=\frac{I_{0}}{u_{0} \pi r_{b}^{2}}$ & $6.78 \times 10^{-3}\left(\mathrm{C} / \mathrm{m}^{3}\right)$ \\
dc beam charge density & $\omega_{p}=\sqrt{\frac{e \rho_{0}}{m_{e} \epsilon_{0}}}$ & $1.16 \times 10^{10}(\mathrm{rad} / \mathrm{s})$ \\
plasma frequency & & \\
effective plasma & $\omega_{q}=\sqrt{R_{\mathrm{sc}}} \omega_{p}$ & $1.16 \times 10^{9}(\mathrm{rad} / \mathrm{s})$ \\
frequency & $\lambda_{p}=\frac{2 \pi u_{0}}{\omega_{p}}$ & $2.48(\mathrm{~cm})$ \\
plasma wavelength & & \\
effective plasma & $\lambda_{q}=\frac{\lambda_{p}}{\sqrt{R_{\mathrm{sc}}}}$ & $24.84(\mathrm{~cm})$ \\
wavelength &
\end{tabular}

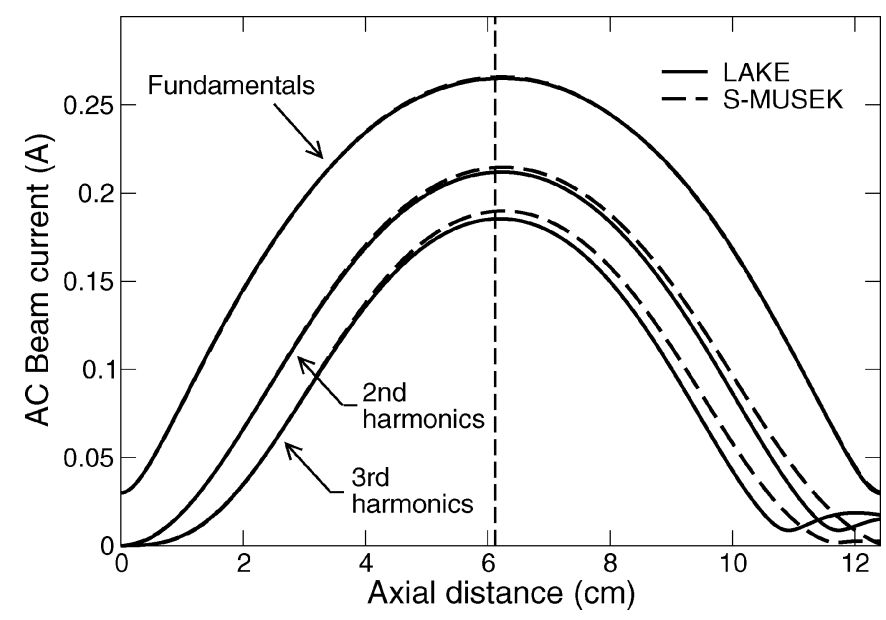

Fig. 1. Beam current envelopes for LAKE and S-MUSEK simulations at the fundamental, second, and third harmonics for input modulation $\epsilon_{a}=0.2, \varphi_{a}=$ $0^{\circ}$. The dashed vertical line is located at $z=\lambda_{q} / 4$.

\section{A. Simulation Comparison}

Before giving analytic results we benchmark numerical solutions of the S-MUSEK and LAKE models of [19] against each other. We choose a drive frequency of $f_{a}=1848 \mathrm{MHz}$ and an input modulation of $\epsilon_{a}=0.2, \varphi_{a}=0^{\circ}$. In Fig. 1 we show the beam current envelopes at the fundamental, second harmonic, and third harmonic for a length of $z=\lambda_{q} / 2$. Consistent with the neglect of some nonlinearity in deriving S-MUSEK, we see that the deviations between the models increase for higher order harmonics. Although Fig. 1 only shows up to the third harmonic, the S-MUSEK and LAKE simulations compute the evolution of harmonics up to the tenth order. Including higher orders of harmonics in the S-MUSEK simulations draws the results closer to the LAKE results. However, we consider the agreement in Fig. 1 sufficient for our purposes.

In Fig. 2 we show the beam current versus axial position for an instant of time predicted by LAKE. The current is computed using the formula

$$
I(z, t)=\sum_{j} \frac{I_{0 j}}{\left|\frac{\partial \Psi_{j}}{\partial \psi_{0 j}}\right|}
$$




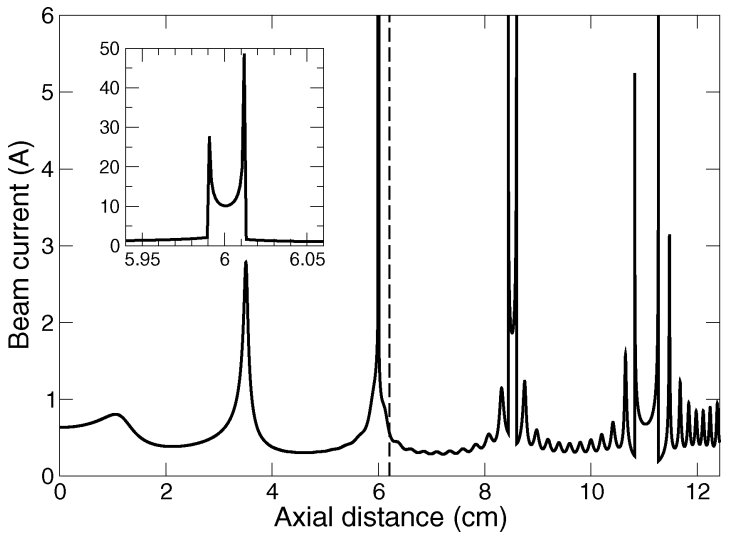

Fig. 2. Beam current versus axial distance predicted by LAKE for the case of Fig. 1. The expanded view around $z=6.0 \mathrm{~cm}$ shows the two-peaked structure of the current providing confirmation that electron overtaking occurs before $z=$ $\lambda_{q} / 4$.

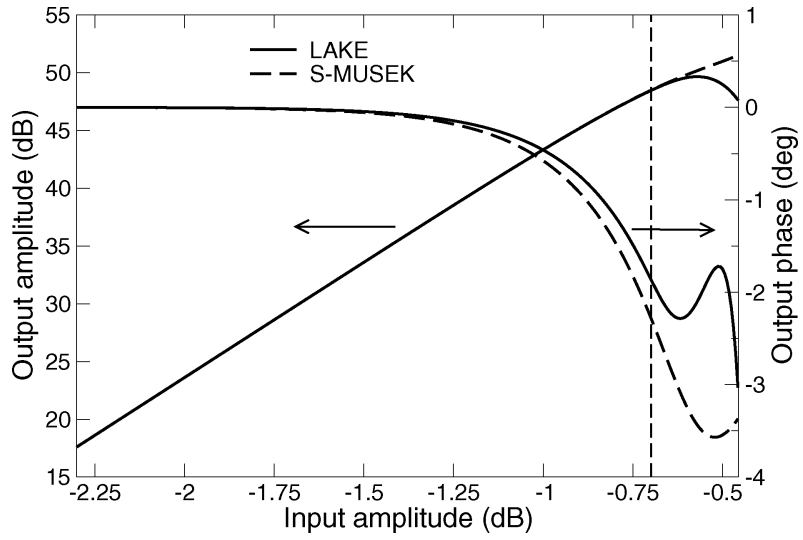

Fig. 3. AM/AM and AM/PM curves predicted by LAKE and S-MUSEK. The $x$ axis is a decibel measure of the velocity modulation $\log \epsilon_{a}$, and the left-hand $y$ axis is a decibel measure of the fundamental beam current $20 \log \left(\left|\tilde{i}_{1}\right| / 10^{-3}\right)$. The dashed vertical line represents $\epsilon_{a}=0.2$ and corresponds to about $1.2 \mathrm{~dB}$ of gain compression.

where the sum is over all disks $j$ that have the same $(z, t)$ coordinates, $\Psi_{j}(z)=z-t_{j}(z)$, and $\psi_{0 j}=\Psi_{j}(0)$. In Fig. 2 we also show an expanded view of the current waveform around $z=6.0 \mathrm{~cm}$. The two peaked structure confirms the multivalued nature of the current. Furthermore, we see that the overtaking becomes more pronounced beyond $z=8 \mathrm{~cm}$, supporting the fact that the deviation of the models increases beyond $z=8 \mathrm{~cm}$ due to the inability of S-MUSEK to model electron overtaking.

Lastly, we show in Fig. 3 AM/AM and AM/PM curves 2 predicted by the two models. The dashed vertical line in Fig. 3 represents the $\epsilon_{a}=0.2$ input level which generated Figs. 1 and 2 , and corresponds to about $1.2 \mathrm{~dB}$ of gain compression. We see that in the "linear gain regime" S-MUSEK and LAKE simulations agree almost exactly in predicted output amplitude, while there is a slight difference in the predicted output phase. Differences in the output phase predictions for $\epsilon_{a}<0.2$ are attributed to the small nonlinearities neglected in deriving S-MUSEK. However, the agreement is close enough

\footnotetext{
${ }^{2}$ For the "AM/PM curve" what is actually shown is fundamental output current phase, not the "conversion coefficient," or derivative of the output phase with respect to the input power. We will loosely refer to the output phase curve as the AM/PM curve.
}

for $\epsilon_{a}<0.2$ to confirm that the approximate S-MUSEK model captures the important nonlinear distortion physics of the more accurate LAKE model. Since we are putting aside the details of coupling to input and output cavities, there is not a clear choice for what units to use for input and output modulation levels. For the input we have chosen a decibel measure of the input amplitude $\log \epsilon_{a}$, and for the output we have chosen a decibel measure of the amplitude of the fundamental beam current modulation $\tilde{i}_{1}, 20 \log \left(\left|\tilde{i}_{1}\right| / 10^{-3}\right)$. In the next section we will compute these curves analytically, and explain the amplitude and phase distortion using nonlinear space charge waves.

\section{B. Analytic Results}

With the simulation comparisons of the previous section we can now confidently compare the S-MUSEK analytic solution to the S-MUSEK simulation and know that the results have been verified by a large signal code.

While analytic solutions for the Eulerian model were first worked out in a TWT [11], [12], there are important physics differences between a TWT and a modulated drifting electron beam that are manifest in the following analytic solutions. The TWT works on the principal of exponential growth, whereas the modulated electron beam in a klystron does not. The analytic solution to both the S-MUSE model for a TWT and the S-MUSEK model in this paper are an infinite series of complex exponential modes [11]. In practical cases, of course, one hopes that the first several terms in the series provide an adequate approximation to the real solution. While this is very much the case in the TWT, i.e., a very few growing exponential terms dominate the solution near the output end of the TWT [11], it is not necessarily the case for the modulated electron beam equations. We have found for the modulated beam one is required at times to keep a very large number of complex exponential modes to adequately approximate the solutions found by simulations.

We have implemented the analytic solution to S-MUSEK in a MATHEMATICA notebook. Due to the required sophistication of programming the general solution [11], and the amount of effort it would take to implement this programming, we typically do not make an attempt in the program to combine modes with identical exponents, nor do we properly compute the secular modes that arise in the solution of (5) [11]. The results shown next confirm that our methods are reasonable for the cases considered. No effort has been made to try and reduce the solution down to a minimal set of modes, as this seems like an ambitious task that will perhaps produce only little added physical insight.

In the results comparing the analytic and simulation predictions of S-MUSEK, we compare beam charge density modulations rather than beam current modulations. The reason for such a comparison is that beam charge density is a state variable of the S-MUSEK formulation, rather than a nonlinear product of state variables, i.e.

$$
\tilde{i}_{\ell}=S \sum_{\substack{m, n \\ f_{m}+f_{n}=f_{\ell}}} \tilde{\rho}_{m} \tilde{v}_{n}
$$

where $S$ is the electron beam cross sectional area. Since the beam density typically dominates the beam current, especially at $z=\lambda_{q} / 4$ where the density modulation is maximum and the 


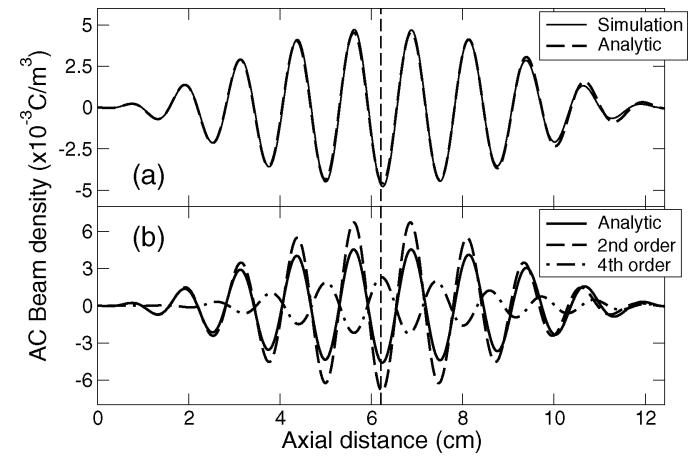

Fig. 4. (a) Simulation and analytic solution of beam density at the second harmonic. (b) Second- and fourth-order components that sum to make the total analytic solution. The second-order solutions only account for mixing of the drive signals. The fourth-order solutions account for mixing of the drive signals, as well as mixing of the third-order products (third harmonic and fundamental $f=2 f-f$ ) with the drive signals. The input level is $\epsilon_{a}=0.2$.

velocity modulation is minimum, one can assume that the beam current solution closely follows the beam density solution.

1) Harmonics: The comparison of the beam density modulations from the analytic solution and S-MUSEK simulation at the second harmonic is shown in Fig. 4. In Fig. 4(a) we show the analytic solution compared to the S-MUSEK simulation, which confirms nearly identical agreement between the analytic solution and the simulation. In Fig. 4(b) we show the "second order" and "fourth order" components of the solution which sum to create the analytic solution. The second-order solutions account for mixing of the drive signals, and the fourth-order solutions account mixing of the third-order products (third harmonics and fundamental $f=2 f-f$ ) with the drive signals. As an example of the number of required modes to predict the solutions, for the sum frequency there are 12 modes for the second-order solution, and 903 modes for the fourth-order solution, which includes degenerate modes, i.e., modes with identical complex exponents.

2) Fundamental: For small inputs the current density modulation is described by the linear space charge waves. However as the input level is increased beyond a certain level there is a gain compression, or amplitude distortion, phenomenon as seen in Fig. 3, and the linear space charge waves predict larger density modulations than the nonlinear models exhibit. Since the fundamental is a "self-intermodulation" product, i.e., $f=2 f-f=3 f-2 f$, the analytic solution at the fundamental consists of complex exponential modes due to odd-order intermodulation products (third, fifth, etc.). In Fig. 5 we show simulation and analytic prediction of the AM/AM and AM/PM curves accounting for only the linear and self-3IM contributions. The agreement indicates that the complex exponential modal solution, i.e., nonlinear space charge waves, is an accurate picture of the physics, at least up to $1.2 \mathrm{~dB}$ of gain compression. We suspect that accounting for the self-5IM contribution would produce better agreement between the phase predicted by the analytic solution and simulation, as is the case in the TWT [12], but we have not done the computation due to the very large number of modes required for the self-5IM contribution.

In Fig. 6 we show the simulation and analytic solution for the beam density at the fundamental with $\epsilon_{a}=0.2$. Fig. 6(a) shows that the analytic solution and simulation agree very well. In Fig. 6(b) we show the "linear" and "3IM" contributions which

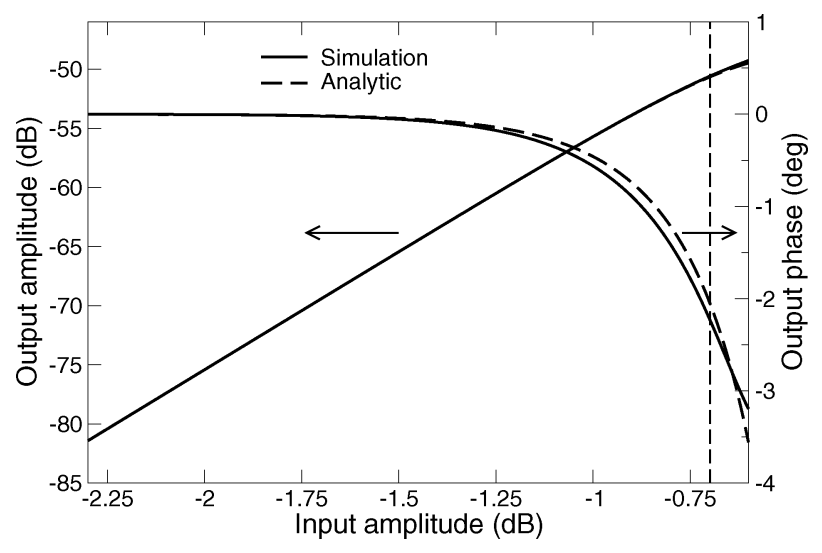

Fig. 5. S-MUSEK simulation and analytic solution for AM/AM and AM/PM curves. Shown are the amplitude and phase of the fundamental beam density modulation, whereas in Fig. 3 the amplitude and phase of the beam current modulation at the fundamental are shown. The dashed vertical line represents $\epsilon_{a}=0.2$

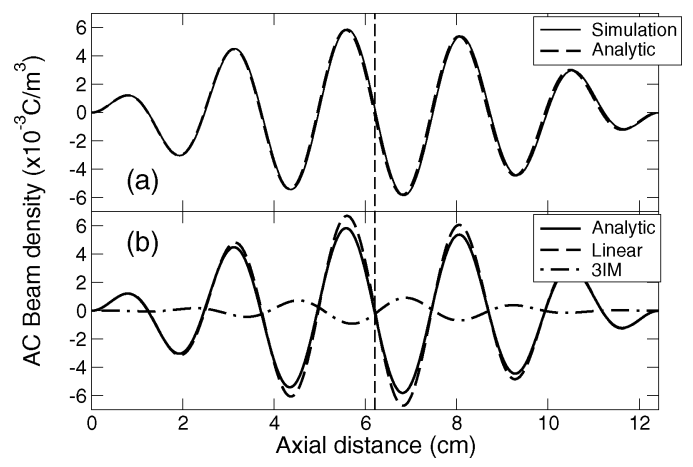

Fig. 6. (a) Simulation and analytic solution for the beam density at $1848 \mathrm{MHz}$ (b) The linear and 3IM contributions that sum to make the total analytic solution. The input level is $\epsilon_{a}=0.2$.

sum to make the total analytic solution. One sees that the 3IM contribution is out of phase with the fundamental and therefore accounts for the reduction in output level, or gain compression. Moreover, since the $3 \mathrm{IM}$ contribution is not precisely $180^{\circ}$ out of phase with the linear contribution, the resulting phase of the waveform will be slightly changed from the linear space charge waves. For larger input powers the 3IM contribution is a larger component of the solution and will push the phase further from that of the linear space charge waves, as seen in the AM/PM curves of Figs. 3 and 5.

\section{TWO-FREQUENCY INPUT}

When a klystron is driven by two narrowly spaced carrier frequencies the nonlinearity of the electron beam generates beam modulations at the so-called intermodulation frequencies. For example, for drive frequencies $f_{a}$ and $f_{b}$ the largest intermodulation products near the drive frequencies are the $3 \mathrm{IMs} 2 f_{b}-f_{a}$ and $2 f_{a}-f_{b}$. The generation of these distortions is undesirable since it degrades communications using the carriers. It has been shown experimentally and numerically for steady state inputs [4] that by including the intermodulation frequency as an input with proper amplitude and phase, that the intermodulation frequency can be canceled in the output. Furthermore, for nonsteady state inputs as found in communications, injection of the 


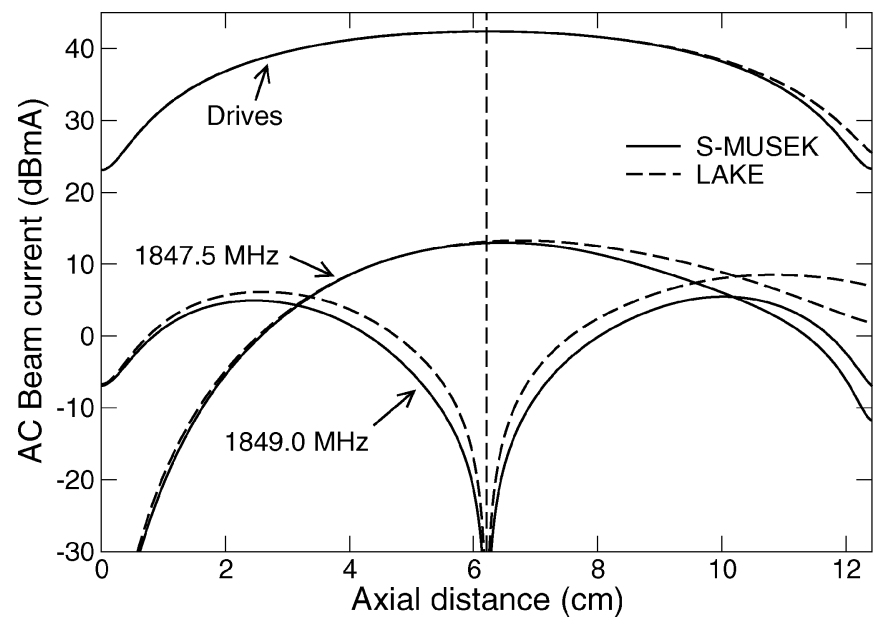

Fig. 7. Beam current envelopes for drive signals and 3IMs for the case of 3IM injection to cancel the 3IM. The injected 3IM is $2 f_{b}-f_{a}=1849 \mathrm{MHz}$. The drive signals for both models have $\epsilon_{a}=\epsilon_{b}=0.095$ and $\varphi_{a}=\varphi_{b}=0^{\circ}$. The inputs for the injected 3IM are for S-MUSEK $\epsilon_{2 f_{b}-f_{a}}=3.0027 \times 10^{-3}$, $\varphi_{2 f_{b}-f_{a}}=-16.97^{\circ}$ and for LAKE $\epsilon_{2 f_{b}-f_{a}}=3.0695 \times 10^{-3}, \varphi_{2 f_{b}-f_{a}}=$ $-12.45^{\circ}$.

third-order intermodulation spectrum is the basis for predistortion linearizers [18].

For comparison with [4] we consider two closely spaced input frequencies $\left(f_{a}=1848 \mathrm{MHz}, f_{b}=1848.5 \mathrm{MHz}\right)$, the second harmonics and sum frequency $\left(2 f_{a}=3696 \mathrm{MHz}, f_{a}+f_{b}=\right.$ 3696.5 MHz, $\left.2 f_{b}=3697 \mathrm{MHz}\right)$, the 3IM products $\left(2 f_{a}-f_{b}=\right.$ 1847.5 MHz, $2 f_{b}-f_{a}=1849 \mathrm{MHz}$ ), and cancellation of the 3IM $2 f_{b}-f_{a}$ with an injected modulation at the intermodulation frequency. The amplitude of the velocity modulations $\epsilon_{a, b}=0.095$ are set such that the square of the 3IM beam current modulation, which is proportional to cavity power, is about 30-dB down from the fundamental at $z=\lambda_{q} / 4$ as in [4]. The input phases are set to zero to be consistent with [4]. We choose the base frequency as $\omega_{0} / 2 \pi=5 \times 10^{5} \mathrm{~Hz}$, so that the drive frequencies have angular frequencies of $3696 \omega_{0}$ and $3697 \omega_{0}$ respectively, and the 3IM of interest has angular frequency $3698 \omega_{0}$.

\section{A. Simulation Comparison}

We have performed benchmarking of the numerical solutions S-MUSEK and LAKE for the two-frequency input similar to Section III-A. In general, the agreement between the models in predicting beam current for drive frequencies, sum and harmonic frequencies, and 3IM's is comparable to the agreement seen in Section III-A. This should be expected since the total amplitude modulation when the two inputs add in phase is $2 \epsilon_{a, b}=0.19$, which is slightly smaller than the value $\epsilon_{a}=0.2$ used in Section III-A. For the two-frequency input we again find that there is a small amount of electron overtaking at $z=5.99 \mathrm{~cm}$.

The only results from the simulation benchmarking we show are for the case of 3IM injection to cancel the 3IM. An additional input modulation at $1849 \mathrm{MHz}$ is added where the amplitude and phase is set such that the beam current modulation is canceled at $z=\lambda_{q} / 4$. The results predicted by S-MUSEK and LAKE showing the fundamentals and the 3IMs are shown in Fig. 7. Due to approximations in deriving S-MUSEK, and

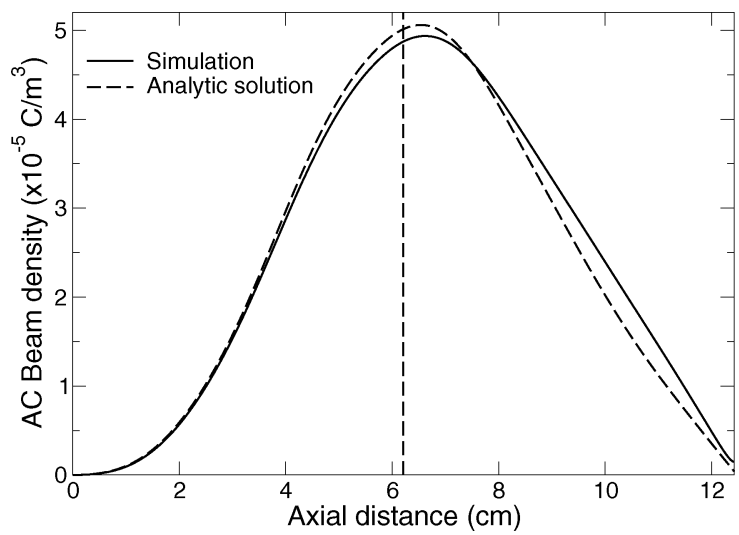

Fig. 8. Beam charge density envelopes at $1849 \mathrm{MHz}$ predicted by the analytic solution and simulation. The inputs are the same as those in Section IV-A.

the fact that there is a small amount of electron overtaking, the input amplitude and phase of the injected 3IM differs quantitatively between the two models, although the predicted quantitative outputs are again seen to be in good agreement. The simulations account for up to third-order intermodulation products.

In this case, and the case treated in Section IV-B, the cancellation is essentially perfect, i.e., by carefully choosing the inputs the beam modulations at the 3IM can be made to be as close to zero as desired. Therefore, it is not really appropriate to discuss the level of 3IM suppression obtained in decibels. In an experiment of course one never achieves this perfect cancellation, and it is entirely appropriate to consider the amount of suppression obtained. In [4] the authors were able to reduce the 3IM level by $25 \mathrm{~dB}$.

\section{B. Analytic Solution}

First we look at results for the 3IM frequency before and after cancellation, then the solutions at the fundamental, and finally the solutions at the second harmonics and the sum frequency.

1) Intermodulation Frequencies: In Fig. 8 we show the simulation and analytic predictions of the nonlinearly generated beam density envelopes at the 3IM frequency. The number of modes required to predict this solution is 201 , where this includes some degenerate modes.

For the analytic solution of the 3IM signal with 3IM injection we are able to, to a good approximation, superpose the nonlinearly generated 3IM shown in Fig. 8 with a linear solution (space charge waves) for a small amplitude injected signal at the 3IM frequency. The two signals then superimpose to cancel at $z=\lambda_{q} / 4$. We show the composite waveforms from simulation and the analytic solution in Fig. 9. The phase differences between the signals is due to our neglecting some third-order and higher order modes in the analytic solution, which then requires a change in input phase to get zero beam density at $z=\lambda_{q} / 4$. The simulation inputs are changed slightly from Fig. 7 in order to have the 3IM beam density modulation cancel at $z=\lambda_{q} / 4$ rather than the 3IM beam current modulation.

Technically, the introduction of the injected 3IM generates even more intermodulation frequencies than are present with two-frequency injection as it is considered as an additional drive signal [11]. However, in computing the solutions in Fig. 9 we have ignored the additional modes that are actually generated, 


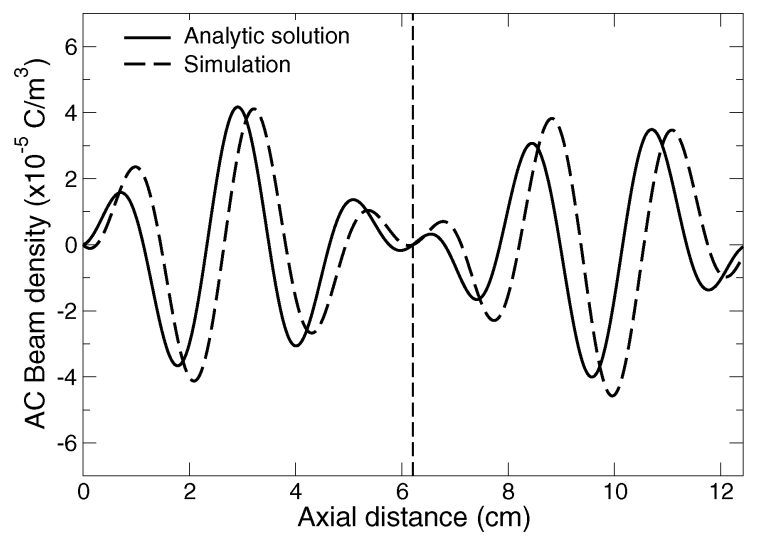

Fig. 9. Simulation and analytic solutions to beam density modulations at 1849 MHz with 3IM injection to cancel the 3IM. The inputs for the injected 3IM are for the simulation $\epsilon_{2 f_{b}-f_{a}}=3.1153 \times 10^{-3}, \varphi_{2 f_{b}-f_{a}}=-16.11^{\circ}$ and for the analytic solution $\epsilon_{2 f_{b}-f_{a}}=2.957 \times 10^{-3}, \varphi_{2 f_{b}-f_{a}}=-10.77^{\circ}$.

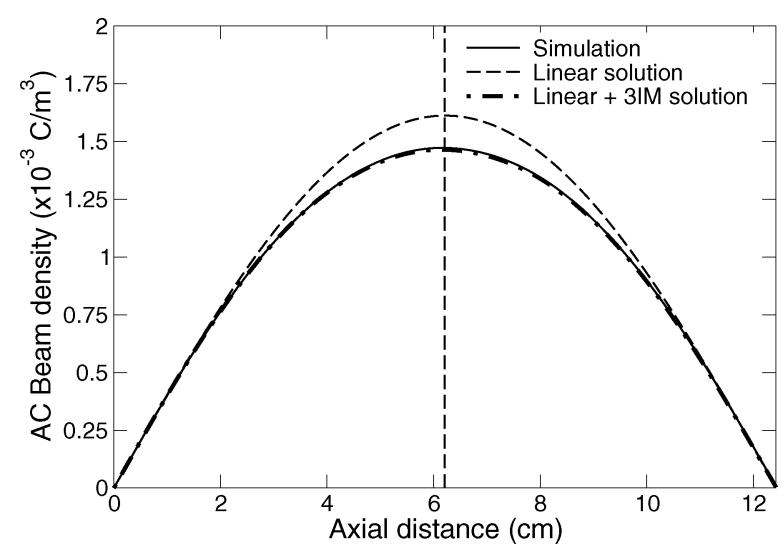

Fig. 10. Beam density envelopes at $1848 \mathrm{MHz}$. Shown are simulation, the linear contribution to the analytic solution, and the analytic solution accounting for the linear contribution and the 3IM contribution.

and this may partially account for quantitative differences seen. We will see that the additional modes due to the injected 3IM play a larger role in determining the levels of the harmonics.

2) Drive Signals: Next we consider analytic solutions for the drive frequencies for the case of no 3IM injection. In Fig. 10 we compare the amplitudes of the $1848 \mathrm{MHz}$ drive signal simulation, the linear solution, and the analytic solution when one accounts for the third-order intermodulation at the fundamental frequency, i.e., $f_{a}=2 f_{a}-f_{a}=f_{a}+f_{b}-f_{b}$. Note that compared to the single-frequency input case in Section III-B there is an additional 3IM mode at the fundamental due to the presence of the other drive signal. The phasing of the 3IM contribution again accounts for a gain compression relative to linear behavior. The number of modes used in predicting the intermodulation effect was 327, which includes degenerate modes. The results for the other drive frequency $1848.5 \mathrm{MHz}$ are qualitatively the same as those shown in Fig. 10.

3) Harmonics: The comparison of the beam density modulation envelopes from the analytic solution and S-MUSEK simulation at the second harmonics and sum frequency are shown in Fig. 11 for the case where there is no injected 3IM. We show both "second-order solutions" and "fourth-order solutions." The second-order solutions account only for mixing of the drive signals. The fourth-order solutions account for mixing of the drive

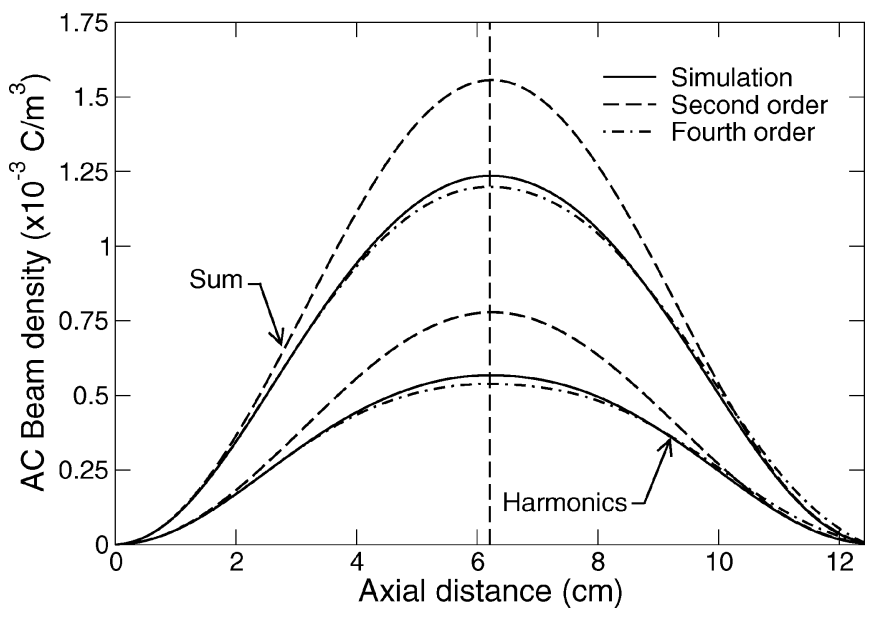

Fig. 11. Simulation and analytic solution of beam density at second harmonics and sum frequency for case of two-frequency excitation. The second-order solutions only account for mixing of the drive signals. The fourth-order solutions account for mixing of the drive signals, as well as mixing of the third-order products (third harmonics, 3IMs, and drives) with the drive signals. Note that both second harmonics are shown, but they are indistinguishable due to the narrow drive frequency spacing.

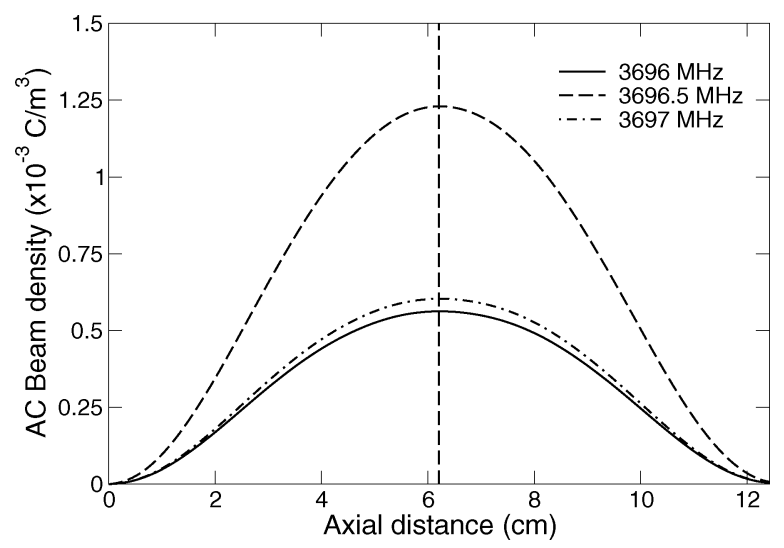

Fig. 12. Simulations of beam density envelopes at second harmonics and sum frequency for the case of 3IM injection. The second harmonic at $3697 \mathrm{MHz}$ is enhanced due to the nonlinear mixing of the injected 3IM at $1849 \mathrm{MHz}$ and the drive signal $1848 \mathrm{MHz}$.

signals, as well as mixing of the third-order products (third harmonics, 3IMs, and drives) with the drive signals. The number of modes required to predict the sum frequency is 21 for the second-order solution, and 7347 for the fourth-order solution, which includes degenerate modes.

In Fig. 12 we show the harmonic and sum frequency envelopes from simulation when the 3IM is injected. The second harmonic of $1848.5-3697 \mathrm{MHz}$, is seen to be larger than the second harmonic $3696 \mathrm{MHz}$ (in Fig. 11 the two harmonics are indistinguishable). This is due to the fact that $3697 \mathrm{MHz}$ is for this case not only the second harmonic of $f_{b}$, but also a second-order product formed between the injected 3IM, $2 f_{b}-$ $f_{a}$, and the drive signal $f_{a}$. We did not compute the analytic solution for this case.

\section{CONCLUSION}

We presented insights into the modal physics of the nonlinear behavior in a modulated electron beam as found in a klystron 
amplifier using an analytic nonlinear space charge wave solution to a nonlinear Eulerian model. For a single-frequency input the mechanism for amplitude and phase distortion was the generation of "self-intermodulation" distortions at the fundamental, e.g., $f=2 f-f$. The self-3IM contributions add out of phase with the linear space charge waves to produce gain compression and phase distortion. Compared to a conventional large-signal code, this view is shown to be valid up to $1.2 \mathrm{~dB}$ of gain compression. We speculate that the description applies into saturation as well, although this can not be proven with the current formulation of S-MUSEK. It is possible that a spectral analysis of the techniques found in [15] could be used to prove the conjecture.

For a two-frequency input nonlinear space charge waves describe the generation of the intermodulation frequencies, and modification of the amplitude and phase of the drive signals due to intermodulations at the drive frequencies. Furthermore, to accurately describe the evolution of second harmonic beam modulations one needs to account for second-order contributions (mixing of the linear space charge waves) as well as fourthorder contributions (mixing of third-order contributions with the linear space charge waves). Lastly, for inputs meant to replicate conditions in [4], cancellation of the intermodulation frequency by injecting a signal at the same frequency is approximately modeled by superposition of the nonlinearly generated 3IM frequency with linear space charge waves due to the injection.

\section{APPENDIX}

As in the case of the TWT the S-MUSEK model of the modulated electron beam can be written in a vector form that is amenable to analysis [9], [11]. Since computation of the general analytic solution follows nearly identically to [11, App. I], except for differing matrix and tensor entries which may be found in [19, App. E], we will not repeat this general solution here. We will however state certain results and point out salient features of the results.

We use the vector notation of [19] where $\mathbf{x}_{\ell}=$ $\left[\mathbf{x}_{\ell_{1}} \mathbf{x}_{\ell_{2}} \mathbf{x}_{\ell_{3}}\right]^{\mathrm{T}}=\left[\tilde{E}_{\ell} \tilde{v}_{\ell} \tilde{\rho}_{\ell}\right]^{\mathrm{T}}$. Due to the Fourier series used in the model, for each positive frequency $f_{\ell}$ there is a corresponding negative frequency indexed by $-\ell$ with $f_{-\ell}=-f_{\ell}$. Furthermore $\mathbf{x}_{-\ell}=\mathbf{x}_{\ell}^{*}$. The differential equation for $\mathbf{x}_{\ell}$ is

$$
\dot{\mathbf{x}}_{\ell}=\mathbf{A}_{\ell} \mathbf{x}_{\ell}+\sum_{\substack{m, n \\ f_{m}+f_{n}=f_{\ell}}} \mathbf{H}_{\ell m n}\left(\mathbf{x}_{m}, \mathbf{x}_{n}\right)
$$

where matrix and tensor components $\mathbf{A}_{\ell_{i j}}$ and $\mathbf{H}_{\ell_{i} m_{j} n_{k}}$ are given in [19]. One can show that (2) may be solved with a series solution

$$
\mathbf{x}_{\ell}=\sum_{\alpha=1}^{\infty} \mathbf{x}_{\ell}^{(\alpha)}
$$

and that this series converges under the appropriate conditions. The index $\alpha$ is related to the order of intermodulation product [10]. Substituting (3) into (2) gives the differential equations for the series terms

$$
\dot{\mathbf{x}}_{\ell}^{(1)}=\mathbf{A}_{\ell} \mathbf{x}_{\ell}^{(1)} \quad \mathbf{x}_{\ell}(0)=\mathbf{w}_{\ell}
$$

$$
\begin{aligned}
\dot{\mathbf{x}}_{\ell}^{(\alpha)} & =\mathbf{A}_{\ell} \mathbf{x}_{\ell}^{(\alpha)}+\sum_{\beta=1}^{\alpha-1} \sum_{\substack{m, n \\
f_{m}+f_{n}=f_{\ell}}} \mathbf{H}_{\ell m n}\left(\mathbf{x}_{m}^{(\beta)}, \mathbf{x}_{n}^{(\alpha-\beta)}\right) \\
\mathbf{x}_{\ell}^{(\alpha)}(0) & =\mathbf{0} \quad \alpha=2,3,4, \ldots
\end{aligned}
$$

where $\mathbf{w}_{\ell}$ is the initial data vector. Note that the quadratic nonlinearities of S-MUSEK are represented by the tensor $\mathbf{H}_{\ell m n}$. In general the solution of (5) may be written as a sum of complex exponential modes

$$
\mathbf{x}_{\ell}^{(\alpha)}=\sum_{p=1}^{N_{\ell}^{(\alpha)}} \mathbf{a}_{\ell}^{(\alpha)[p]} e^{i \kappa_{\ell}^{(\alpha)[p]} z} .
$$

To compute the mode amplitudes $\mathbf{a}_{\ell}^{(\alpha)[p]}$ and wavenumbers $\kappa_{\ell}^{(\alpha)[p]}$ we note that interested readers may formulate a theory analogous to [11, App. I].

The $\alpha=1$ (linear) solution of S-MUSEK is the solution of (4). The eigenvalues of the matrix $\mathbf{A}_{\ell}$, combined with the complex phase factor $e^{i f_{\ell} \omega_{0} \psi}$, produce the conventional fast and slow space charge waves [16], [17]. The linear solution also generates a third complex exponential mode in addition to the two space charge waves since our model equations use a differential equation to relate $E$ to $\rho$, where conventional theories use an algebraic relation between $E$ and $\rho$.

Second-order solutions, i.e., second harmonics, sum and difference frequencies, are the solution of (5) with $\alpha=2$. In this equation $\mathbf{H}_{\ell m n}$ nonlinearly mixes the frequencies creating the product frequency. In the case of a second harmonic a driving frequency experiences "self mixing," whereas in a sum or difference frequency two driving frequencies are mixed. The number of complex exponential modes in the solution of (5) is depends on the number of frequencies mixing to produce the result. In the case of sum and difference frequencies the quadratic nonlinearity produces nine modes (outer product of sums of three complex exponentials), each of which appear twice due to the sum over $\beta$. These modes are then combined with the three "natural modes" of the linear part of (5), producing a total of 12 possible modes. Details of this mode generation can again be gleaned by studying the general solution in [11, App. I].

Third-order solutions, e.g., third harmonics and third-order intermodulation frequencies, are the solution of (5) with $\alpha=3$. In this equation $\mathbf{H}_{\ell m n}$ nonlinearly mixes the drive frequencies with the second-order solutions to create the product frequency. As seen in Sections III-B and IV-B the 3IM effect at the drive frequency plays a large role in determining the modulation amplitude and phase at the drive frequency. For two input frequencies $f_{a}$ and $f_{b}$ the number of possible third-order modes at $f_{a}$ becomes large quickly since there are three first and second-order pairs that can mix to produce $f_{a}$, i.e., $\left(2 f_{a}, f_{a}\right),\left(f_{b}+f_{a}, f_{b}\right)$, and $\left(f_{b}-f_{a}, f_{b}\right)$. For example, $\left(f_{b}+f_{a}, f_{b}\right)$ produces a possible 36 modes, including degenerate modes due to the structure of solutions to (4) and (5) for $\alpha=2$.

It is important to place our analytic solution in the context of earlier analytic theories of a modulated electron beam, e.g., [7], [8], [23]. The Eulerian formulation of S-MUSEK does not allow multivalued solutions, which might limit the S-MUSEK solution usefulness in some situations. In contrast, the theories in [7] and [8] use Lagrangian coordinates and hence give predictions beyond overtaking "...although the theory is not strictly valid after electron overtaking has occurred" [7]. However, this 
might not be a severe limitation since, to maximize efficiency, most klystrons are designed and driven such that overtaking does not occur, or only a small amount of overtaking occurs [24]. We show in Sections III and IV that in a case where electron overtaking is "weak," that the S-MUSEK theory provides useful results.

The modal structure of the S-MUSEK solution has clear advantages over other theories in applications such as that given in this paper. The S-MUSEK solution gives a clear physical picture of how driven modes can combine with nonlinear space charge modes to achieve cancellation of the intermodulation frequencies. Furthermore, modification of the fundamental by the intermodulation process is also clearly seen, as is the modification of second harmonics and sum frequencies by fourth-order contributions. It should be noted that our theory bears some resemblance to the theories of Pashcke, e.g., [23]. However, our formulation is much more general, perhaps since at the time of writing [23] there was no impetus for a general multifrequency formulation.

\section{REFERENCES}

[1] T. Chen, Y. Goren, C. Jensen, P. Lally, and D. Gagne, "A novel technology for linearizing traveling wave tube amplifiers," in MTT-S Int. Microwave Symp. Dig., 2002, pp. 773-776.

[2] M. Wirth, A. Singh, J. Scharer, and J. Booske, "Third-order intermodulation reduction by harmonic injection in a TWT amplifier," IEEE Trans. Electron Devices, vol. 49, no. 12, pp. 1082-1084, Dec. 2002.

[3] J. Qiu, D. Abe, T. Antonsen, Jr., B. Danly, and B. Levush, "Travelingwave tube amplifier performance evaluation and design optimization for applications in digital communications with multilevel modulations," IEEE Trans. Microw. Theory Tech., vol. 51, no. 8, pp. 1911-1919, Aug. 2003.

[4] S. Bhattacharjee, C. Marchewka, J. Welter, R. Kowalczyk, C. Wilsen, Y. Lau, J. Booske, A. Singh, J. Scharer, R. Gilgenbach, M. Neumann, and M. Keyser, "Suppression of third-order intermodulation in a klystron by third-order injection," Phys. Rev. Lett., vol. 90, no. 9, pp. 098303-1 098303-4, 2003.

[5] D. Goebel, R. Liou, W. Menninger, X. Zhai, and E. Adler, "Development of linear traveling wave tubes for telecommunications applications," IEEE Trans. Electron Devices, vol. 48, no. 1, pp. 74-81, Jan. 2001.

[6] D. Abe, B. Levush, T. Antonsen, Jr., D. Whaley, and B. Danly, "Design of a linear C-band helix TWT for digital communications experiments using the CHRISTINE suite of large-signal codes," IEEE Trans. Plasma Sci., vol. 30, no. 6, pp. 1053-1062, Dec. 2002.

[7] S. O. Wallander, "Large signal analytical study of bunching in klystrons," IEEE Trans. Electron Devices, vol. ED-15, no. 8, pp. 595-603, Aug. 1968.

[8] Y. Lau, D. Chernin, C. Wilsen, and R. Gilgenbach, "Theory of intermodulation in a klystron," IEEE Trans. Plasma Sci., vol. 28, no. 3, pp. 959-970, Jun. 2000.

[9] J. Wöhlbier, J. Booske, and I. Dobson, "The multifrequency spectral Eulerian (MUSE) model of a traveling wave tube," IEEE Trans. Plasma Sci., vol. 30, no. 6, pp. 1063-1075, Dec. 2002.

[10] J. Wöhlbier, I. Dobson, and J. Booske, "Generation and growth rates of nonlinear distortions in a traveling wave tube," Phys. Rev. E, Stat. Phys. Plasmas Fluids Relat. Interdiscip. Top., vol. 66, pp. 056504-1-056504-5, 2002.

[11] J. Wöhlbier, J. Booske, and I. Dobson, "On the physics of harmonic injection in a traveling wave tube," IEEE Trans. Plasma Sci., vol. 32, no. 3, pp. 1073-1085, Jun. 2004.

[12] J. Wöhlbier and J. Booske, "Mechanisms for phase distortion in a traveling wave tube," Phys. Rev. E, Stat. Phys. Plasmas Fluids Relat. Interdiscip. Top., vol. 69, pp. 066502-1-066502-16, 2004.

[13] A. Singh, J. Wöhlbier, J. Booske, and J. Scharer, "Experimental verification of the mechanisms for nonlinear harmonic growth and suppression by harmonic injection in traveling wave tubes," Phys. Rev. Lett., vol. 92, pp. 205005-1-205005-4, 2004.
[14] A. Singh, J. Scharer, J. Booske, and J. Wöhlbier, "Second- and thirdorder signal injection for nonlinear distortion suppression in a traveling wave tube," IEEE Trans. Electron Devices, vol. 52, no. 5, May 2005.

[15] X. Li, J. Wöhlbier, S. Jin, and J. Booske, "Eulerian method for computing multivalued solutions of the Euler-Poisson equations and application to wave breaking in klystrons," Phys. Rev. E, Stat. Phys. Plasmas Fluids Relat. Interdiscip. Top., vol. 70, pp. 016502-1016502-122, 2004

[16] W. Hahn, "Small signal theory of velocity modulated electron beams," Gen. Elec. Rev., vol. 42, pp. 258-270, 1939.

[17] S. Ramo, "The electronic-wave theory of velocity modulated tubes," Proc. IRE, vol. 27, pp. 757-763, 1939.

[18] A. Katz, "Linearization: Reducing distortion in power amplifiers," IEEE Microw. Mag., vol. 2, no. 4, pp. 37-49, Dec. 2001.

[19] J. Wöhlbier, "Nonlinear distortion and suppression in traveling wave tubes: Insights and methods Online. Available: http://www.Imsuite.org," Ph.D. dissertation, Univ. Wisconsin, Madison, 2003.

[20] J. Rowe, Nonlinear Electron-Wave Interaction Phenomena. New York: Academic, 1965.

[21] S. Wallander, "Large signal computer analysis of klystron waves," Int. J. Electron., vol. 24, no. 2, pp. 185-196, 1968.

[22] R. Hutter, Beam and Wave Electronics in Microwave Tubes. Princeton, NJ: Van Nostrand, 1960

[23] F. Paschke, "On the nonlinear behavior of electron-beam devices," $R C A$ Rev., vol. 18, pp. 221-242, 1957.

[24] B. Carlsten, private communication, Apr. 2004.

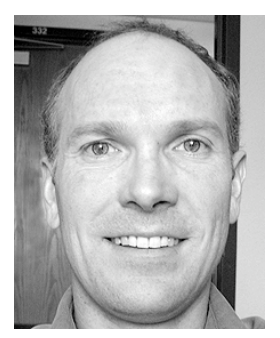

John G. Wöhlbier (M'03) received the Ph.D. degree in electrical engineering from the University of Wisconsin, Madison, in 2003.

In the summer of 2003 he was an Engineering Physics Postdoctoral Scientist at the University of Wisconsin where he worked on ballooning instabilities in three-dimensional plasmas. In 2004, he worked on vacuum device physics as an Agnew National Security Postdoctoral Fellow, Los Alamos National Laboratory. Currently, he is working on the Advanced Simulation and Computing program (ASC) in the Continuum Dynamics Group (CCS-2), Los Alamos. His research interests include plasma physics, fluid dynamics, computational physics, dynamical systems, vacuum electronics, and applied math.

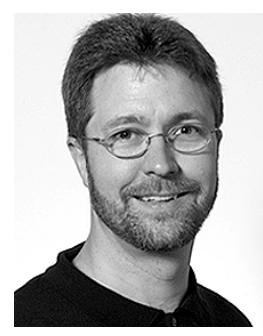

John H. Booske (SM'93) received the Ph.D. degree in nuclear engineering from the University of Michigan, Ann Arbor, in 1985.

He was a Research Scientist with the University of Maryland, College Park, from 1985 to 1989 In 1990, he joined the faculty of the University of Wisconsin, Madison, where he is a Professor of Electrical and Computer Engineering. In 2001, he was appointed Director of the Materials Science Program. His research interests include the experimental and theoretical study of coherent electromagnetic radiation sources and their applications, with emphasis in the RF, microwave, millimeter-wave, and terahertz regimes. Recent research activities include vacuum electronics, microwave and RF heating of materials, and biological/biomedical applications of microwave and RF fields. He is coeditor of the book Microwave and Radio Frequency Applications (Westerville, $\mathrm{OH}$ : American Ceramic Society, 2003) and coeditor of the book Microwave and Millimeter-Wave Power Electronics (Piscataway, NJ: IEEE Press, 2004).

Dr. Booske received the University of Wisconsin Vilas Associate Faculty Award for research excellence and the U.S. National Science Foundation Presidential Young Investigator Award. He has been honored with many teaching awards, including the University of Wisconsin Chancellor's Distinguished Teaching Award and the Benjamin Smith Reynolds Award for Excellence in Teaching Engineering. He served as Co-Director of a U.S. DoD MURI99 Consortium on Innovative Microwave Vacuum Electronics, and as Director of a MURI04 Consortium on cathode field emission and RF window breakdown in high-power microwave sources. He has been a Guest Editor of the IEEE TRANSACTIONS ON Plasma SCIENCE. 